\title{
Tip-enhanced Raman spectroscopy: principles and applications
}

\author{
Naresh Kumar ${ }^{1}$, Sandro Mignuzzi ${ }^{1}$, Weitao $\mathrm{Su}^{2,3}$ and Debdulal Roy ${ }^{1 *}$
}

\author{
* Correspondence: debdulal.roy@ \\ npl.co.uk \\ ${ }^{1}$ National Physical Laboratory, \\ Hampton Road Teddington, \\ Middlesex TW11 OLW, UK \\ Full list of author information is \\ available at the end of the article
}

\begin{abstract}
This review provides a detailed overview of the state of the art in tip-enhanced Raman spectroscopy (TERS) and focuses on its applications at the horizon including those in materials science, chemical science and biological science. The capabilities and potential of TERS are demonstrated by summarising major achievements of TERS applications in disparate fields of scientific research. Finally, an outlook has been given on future development of the technique and the mechanisms of achieving high signal enhancement and spatial resolution.
\end{abstract}

Keywords: Tip-enhanced Raman spectroscopy; Nanotechnology; Nanophotonics

\section{Introduction}

Tip-enhanced Raman spectroscopy (TERS) combines the chemical sensitivity of surface-enhanced Raman spectroscopy (SERS) with high spatial resolution of scanning probe microscopy (SPM) and enables chemical imaging of surfaces at the nanometre length-scale. The concept of TERS was first proposed by Wessel in 1985 [1], and it was experimentally realised in 2000 [2-5]. Since then TERS has rapidly progressed to become a non-destructive scanning probe microscopy tool for surface chemical characterisation [6], and opened up the opportunities to study single macro-molecules with sub-nm spatial resolution [7]. Over the last 15 years, the technique has been used to study scientific problems in biology [8], photovoltaics [9], catalysis [10], semiconductors [11], carbon nanotubes [12], graphene [13] and single molecule detection [7].

Unlike electron spectroscopy and microscopy techniques such as scanning electron microscopy (SEM), transmission electron microscopy (TEM) and X-ray photoelectron spectroscopy (XPS) that require vacuum for their operation, TERS can be used in ambient environment and it is well-suited for the investigation of samples in aqueous mediums $[14,15]$. Although super-resolution fluorescence microscopy techniques can be used for imaging of biological samples below the diffraction limit, the fluorescent labels prevent the observation of samples in their native state [16]. Being a label-free technique, TERS can be used to study the molecules directly particularly for investigating chemical composition and molecular dynamics in biological samples. Therefore, as outlined in the later part of this review, TERS applications on biological samples such as pathogens [17], lipid and cell membranes [18], nucleic acids [19], peptides [20] and proteins [21] have drawn a significant interest. 
This review aims to describe the recent progress in using TERS to obtain novel insights in all the areas of application explored so far. This article is divided into two parts. In the first part, an introduction to TERS principles and instrumentation is presented, and the second part focusses on the applications of TERS in biology, catalysis, polymer-blends, semiconductors, 1-D and 2-D materials and single molecule detection. An attempt has been made to catalogue the significant applications of TERS in diverse areas of scientific research in order to provide a snapshot of the current state-of-the-art.

\section{TERS principles and instrumentation}

The principle of TERS is schematically shown in Fig. 1. A sharp metal or metal-coated SPM tip is positioned at the centre of a laser focus, and the electromagnetic (EM) field at the tip-apex is confined and enhanced due to a combination of localised surface plasmon (LSP) resonance and lightning rod effect. This increase in EM field enhances the Raman signal from the molecules in the vicinity of the tip-apex and enables nanoscale chemical imaging of a surface, overcoming the diffraction limit of SERS and conventional Raman spectroscopy.

\section{Signal enhancement}

Sensitivity and resolution in TERS are mainly interpreted in terms of electromagnetic enhancement mechanism [22], although signal enhancement due to chemical interaction of a TERS tip with the sample has also been reported [23]. The observed EM enhancement at the tip-apex arises from a combination of localised surface plasmon (LSP) resonance and antenna effect that result in a locally confined and enhanced electric field [24], as shown in Fig. 2a. An even greater localised electrical field enhancement is observed at the tip-apex when the LSP wavelength of the tip-apex matches with the wavelength of the excitation laser. Further details of tip-enhancement can be found in the book by S. Kawata and V. M. Shalaev [25].

The LSP wavelength of the TERS tip can be tuned by changing the material, radius or roughness of the tip-apex, which can then be matched with the wavelength of the excitation laser. At resonance, the intensity of observed electrical field at the tip-apex is

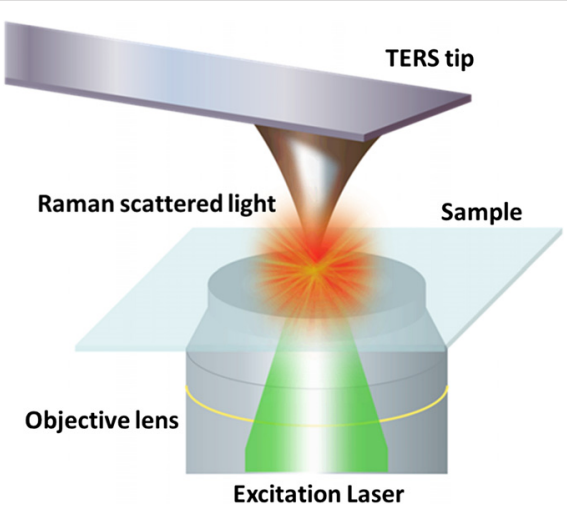

Fig. 1 Schematic diagram of a TERS set-up. A schematic diagram illustrating the principle of AFM-TERS in transmission mode. The LSP resonance at the tip-apex between the tip-LSPs and laser-photons enhances and confines the EM field resulting in the enhancement of Raman signal from the analyte molecules in the immediate vicinity of the tip-apex 


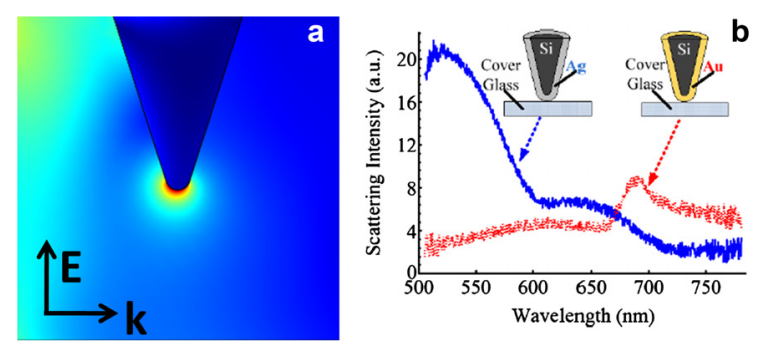

Fig. 2 Antenna effect and plasmon characteristics of silver and gold TERS tips. a Incidence of p-polarised laser ( $k$ indicates the wave vector of the excitation laser) with electric field (E) parallel to the tip-axis at a metal or metal-coated tip causes the EM field intensity to be enhanced and confined at the tip-apex. Red colour represents a higher intensity of EM field. This image is calculated using COMSOL Multiphysics ${ }^{\oplus}$. Tip radius: $15 \mathrm{~nm}$. b Scattering of light from silver- and gold-coated silicon AFM tips. Figure (b) is reproduced by permission of IOP Publishing from reference [26]. Copyright 2013 IOP Publishing. All rights reserved

several orders of magnitude higher than at off-resonance. Although LSP resonance has been observed for many metal nanoparticles, only silver and gold are resonant with lasers in the visible light regime. Generally, the LSP wavelength of a silver TERS tip falls in the blue and green light region, whereas for a gold TERS tip, it falls in the yellow and red light region, as shown in Fig. 2b [26]. However, LSP wavelength is also dependent on the radius of the tip-apex. For example, for a silver tip with a radius smaller than $10 \mathrm{~nm}$, the LSP wavelength may fall in the ultra-violet (UV) region. In principle, such tips can be used to conduct UV-TERS with a lower background signal from the substrate due to the low penetration depth of UV photons [27].

In the near-field of the TERS tip, the intensity of Raman signal from the analyte molecules is proportional to the fourth power of the local electric field. Hence, for nearfield and far-field electric-field intensity $E_{N F}$ and $E_{F F}$ respectively, the enhancement of Raman signal $(\rho)$ is equal to $[22,28]$

$$
\rho=\left(\frac{E_{N F}}{E_{F F}}\right)^{4}
$$

The Raman enhancement factor $(E F)$ in a TERS experiment is calculated using [29]

$$
E F=\left(\frac{I_{\text {Tip-in }}}{I_{\text {Tip-out }}}-1\right) \frac{A_{F F}}{A_{N F}}
$$

where $I_{T i p-i n}$ and $I_{T i p-o u t}$ are the Raman peak intensities measured with the tip in contact and retracted from the sample; $A_{F F}$ is the area of the far-field laser probe; $A_{N F}$ is the effective area of TERS probe, which is usually estimated from the geometric diameter of the tip-apex. The term $\left(\frac{I_{T i p-i n}}{I_{\text {Tip-out }}}-1\right)$ is also described as "contrast".

\section{Experimental set-ups}

Optical geometries of a TERS set-up can be classified into three types: bottom (transmission mode), side, and top illumination, as shown schematically in Fig. 3. Side and top illumination are also referred to as reflection modes. In transmission mode (Fig. 3a), a laser beam is focused onto the sample through a transparent substrate using an objective lens with high numerical aperture (NA), which is usually $>1.0$ [29]. In this geometry, a radially polarised beam provides a tighter focus and stronger longitudinal 


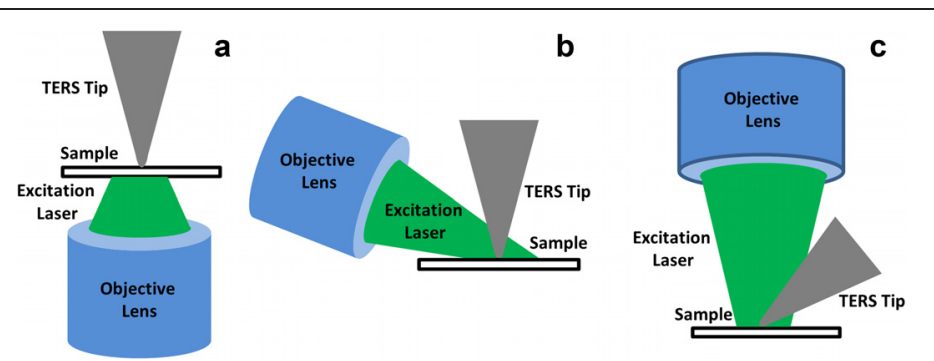

Fig. 3 Schematic diagrams of most commonly used TERS configurations. a Bottom illumination set-up. b Side illumination set-up. c Top illumination set-up

electric field along the tip-shaft compared to linearly polarised beam in the focal place. Theoretical calculations and experimental results have shown that the EM enhancement using the radially polarised beam can be $>4$ times stronger than that of linearly polarised beam [30,31]. Although engineering implementation of the transmission mode set up is simpler, it is limited by the essential requirement of a transparent substrate.

In the side illumination set-up (Fig. 3b), a linearly polarised beam (p-polarisation) [7] is focused onto the tip-apex from the side of the tip via a long working distance objective lens. Because the polarisation is parallel to the tip direction, a strong EM enhancement at the tip-apex can be obtained even with a low NA objective lens. This is especially beneficial for the TERS characterisation of nanoelectronic devices, where substrates are generally non-transparent. In the top illumination set-up (Fig. 3c), the laser is focused from the top onto the end of a tilted STM tip [32] or a nose-type AFM tip [33]. In another type of reflection mode set-up reported by Zhang et al. [34], the laser can also be focused onto the apex of a TERS tip normal to the sample via a parabolic mirror. This geometry has the advantage of being free from chromatic aberration, having a high NA $(\approx 1)$ and a tight laser focus.

\section{Tip preparation}

\section{Metal coating}

It has been nearly 15 years since Zenobi and co-workers used Ag-coated silicon AFM tips to conduct the first TERS measurements [2]. Thermal evaporation in vacuum $\left(10^{-5}-10^{-6} \mathrm{mbar}\right)$ is still the most common method for preparing TERS tips. However, the yield of TERS tips showing large signal enhancement is usually quite low due to the random nucleation and growth process of metal nanoparticles around the tip-apex.

The issue of low yield has been addressed by modifying the refractive index of an AFM tip prior to metal deposition, which leads to improvements in the plasmon resonance characteristics of the TERS tips. Yeo et al. reported a method to prepare Agcoated dielectric TERS tips [35]. A thin $\mathrm{SiO}_{2}, \mathrm{SiO}_{\mathrm{x}}$ or $\mathrm{AlF}_{3}$ film was deposited on the tip surface prior to deposition of the Ag film. Tips prepared by this method were found to have a high EF with a yield close to $100 \%$. Taguchi et al. [36] also obtained almost $100 \%$ yield of TERS tips by oxidising the silicon AFM tips to a thin layer of $\mathrm{SiO}_{2}$ $(\approx 300 \mathrm{~nm})$ before Ag deposition.

Recently, Johnson et al. reported a novel method to prepare reproducible gold TERS tips using a template, which is shown schematically in Fig. 4a [37]. In this process, a 

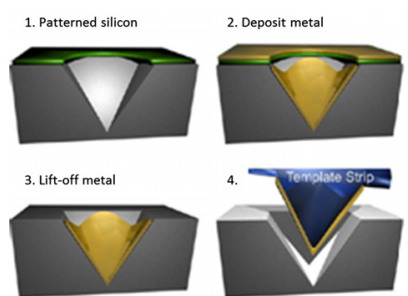

a
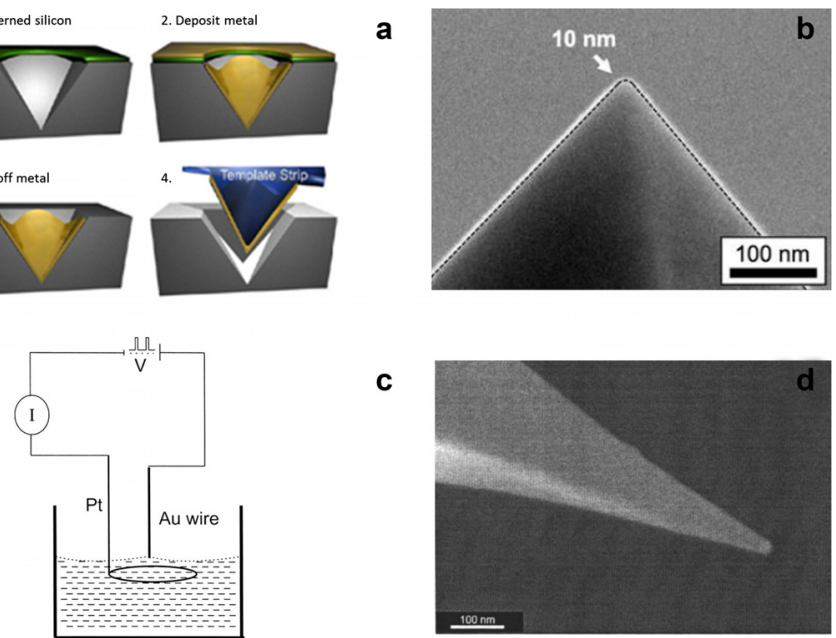

C

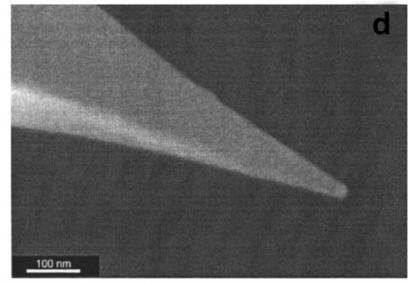

Fig. 4 Preparation of TERS tips by template-stripping and electrochemical etching methods. a Schematic diagram showing the template-stripping method to prepare gold tips. b Scanning electron microscopy (SEM) image of the template-stripped gold tip-apex showing a radius of about $10 \mathrm{~nm}$. c Schematic diagram showing the set-up for etching gold tips. $\mathbf{d}$ SEM image of an etched gold tip. (a) and (b) are reprinted with permission from reference [37]. Copyright 2012 American Chemical Society. (c) and (d) are reprinted with permission from reference [38], Copyright 2013, American Vacuum Society

pyramidal pit is etched on silicon and used as a template to fabricate a gold tip as shown in Fig. 4b. After lift-off from the template, the gold tip can be mounted onto the prong of a quartz tuning fork or a cantilever and used for TERS measurements. Since the size and shape of the pyramidal pits are well-defined and reproducible, enhancement from these tips is more consistent than those prepared by the conventional thermal evaporation method. Because of a thicker layer of metal, the durability of this type of TERS tips is also significantly improved.

\section{Electrochemical etching}

Electrochemical etching methods have been implemented by different research groups to obtain sharp and smooth metal tips [38-40]. For example, in the electrochemical etching of gold tips, a gold wire acts as the anode and a second electrode (gold or platinum) acts as the cathode (as shown schematically in Fig. 4c). Both electrodes are immersed in an etchant and current is passed to etch away the gold wire until the meniscus drops off.

C. Williams and D. Roy [38, 39] used hydrochloric acid as the etchant for etching gold wires according to the following equations

$$
\begin{aligned}
& \mathrm{Au}+4 \mathrm{Cl}^{-} \rightarrow \mathrm{AuCl}_{4}^{-}+3 e^{-} \\
& \mathrm{Au}+2 \mathrm{Cl}^{-} \rightarrow \mathrm{AuCl}_{2}^{-}+e^{-} \\
& \mathrm{AuCl}_{2}^{-}+2 \mathrm{Cl}^{-} \rightarrow \mathrm{AuCl}_{4}^{-}+2 e^{-}
\end{aligned}
$$

Sharp gold tips with radius smaller than $10 \mathrm{~nm}$ (Fig. 4d) can be prepared by this method with high reproducibility. An electrochemical etching method using nitric acid and ethanol to produce sharp, smooth and reproducible silver TERS probes with a tipapex radius of $20 \mathrm{~nm}$ was reported by Lloyd et al. [40]. 


\section{SPM feedback}

TERS systems can be classified into three types according to the different feedback mechanisms used to keep the tip in contact with the surface: contact/tapping mode AFM, shear force and scanning tunnelling microscope (STM), shown schematically in Fig. 5a, b and c, respectively. Since an AFM system is more versatile in terms of usage on both non-conductive and conductive samples, many home-made and commercial TERS systems have adapted the AFM-based TERS setup, first reported by Stockle et al. [2]. In AFM a constant force of interaction is maintained between the cantilever and the sample using a feedback loop. Any deflection of the cantilever due to changes in the force of interaction is measured using a semiconductor laser, which is reflected from the back of an AFM cantilever onto a quadruple photo-detector, as shown in Fig. 5a. The silver or gold-coated AFM tips can usually provide a TERS resolution of $20 \mathrm{~nm}[13,37]$. However, oxidation of silver in ambient air makes the tips lose their enhancing capability within a few hours. Although contact-mode is the most commonly used AFM mode so far, some researchers have also reported strong enhancement of Raman signals using tapping-mode AFM TERS [41-43]. In shear force AFM mode, shown in Fig. 5b, a metal tip is mounted on to the prong of a quartz tuning fork. TERS measurements are conducted by positioning the tip at the centre of the laser focus, and accurately controlling the tip-sample distance using shear-force as the feedback $[44,45]$. In case of STM-TERS, the tip-sample distance is controlled via a feedback loop using the tunnelling current between the tip and sample, as shown schematically in Fig. 5c. STMTERS can be used to measure conductive and semiconductive samples on conductive substrates. High spatial resolutions of $0.5 \mathrm{~nm}$ and $1.7 \mathrm{~nm}$ have been achieved using this scheme in reflection mode geometry [7, 46]. Although most STM-TERS microscope are operated in reflection mode, transmission mode measurements on transparent conductive substrates such as indium tin oxide (ITO) and thin coating $(<20 \mathrm{~nm})$ of metal on glass substrates can be realised.

\section{TERS applications}

Spontaneous Raman spectroscopy has been used as a label-free chemical characterisation tool in materials science [47], chemical engineering [48] and recently in biology [49]. Tipenhanced Raman spectroscopy extends the spatial resolution of the technique to the nanometre length-scale. Attempts have been made to use TERS to study a range of nanostructured materials and biological samples, and have provided insights into the structure and chemistry of these systems that are not possible to obtain by conventional analytical

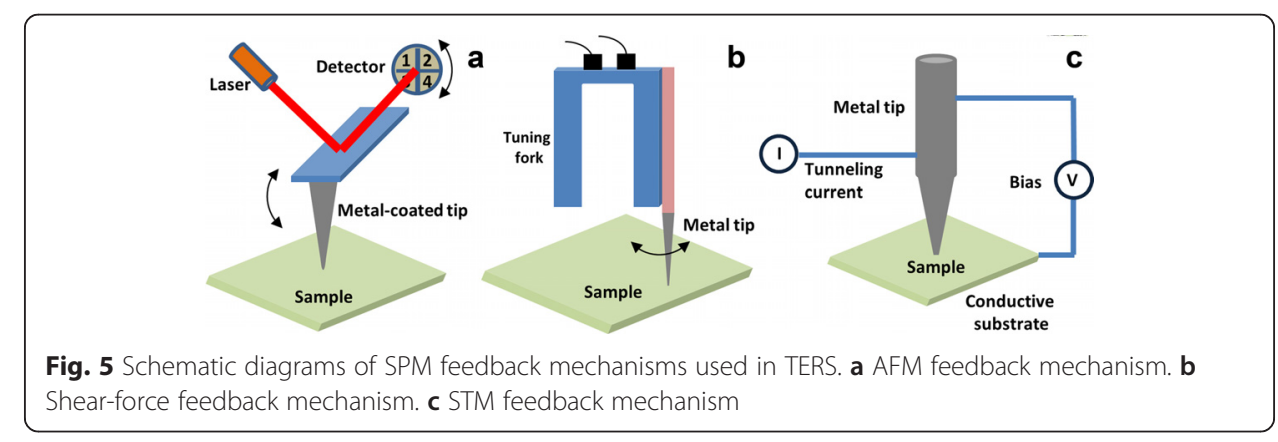


methods. Few specific examples are highlighted here to demonstrate the advancement of the technique.

\section{Pathogens}

Development of target-specific, non-toxic and cost-effective drugs is underpinned by comprehensive understanding of the structure, dynamics and function of pathogens such as bacteria and viruses. TERS can provide label-free, nanoscale chemical information that can be used to measure localised composition of specific components on a pathogen's surface. It can facilitate investigation of biological processes such as host-pathogen interactions, bacterial adhesion, formation of biofilms and bacterial pathogenesis [42]. The first TERS spectrum from a bacterial surface was reported by Neugebauer et al. [42]. In this work, peptides and polysaccharides Raman bands, typical of a bacterial surface could be identified in the near-field spectra with an enhancement factor of $10^{4}-10^{5}$. Furthermore, consecutive TERS spectra measured at the same location showed variation in peak intensity and positions of some bands indicating the protein dynamics at the cell surface induced either by the cellular processes or the interaction with the TERS tip. This demonstrated the feasibility of studying complex biological systems such as bacteria using TERS.

TERS investigation of a virus surface was carried out by Cialla et al. [50]. Raman bands corresponding to the protein coating and ribonucleic acid (RNA) of a single tobacco mosaic virus (diameter $\approx 20 \mathrm{~nm}$ ) were detected in the near-field spectra with a high degree of reproducibility. The authors attributed the variation in the intensity and position of the Raman bands in the near-field to the difference in orientation of the protein and RNA molecules in the vicinity of the TERS tip at different locations on the virus surface.

Wood et al. applied TERS to probe hemozoin crystals in the digestive vacuole of a sectioned malaria parasite-infected single red-blood cell with a spatial resolution of less than $20 \mathrm{~nm}$ [17]. TERS spectra clearly showed the characteristic hemozoin bands consistent with a five-coordinate high-spin ferricheme complex, which could be correlated to a precise position on the crystal by comparison with the AFM topography image. Averaged near-field spectra from different locations on a cell showed reproducibility over a few cells.

The aforementioned studies indicate that TERS can also be used to monitor the interaction of pathogens with their hosts or antibiotics and guide the design of therapeutic drugs that specifically bind to the correct part of the infected cell. Furthermore, the study by Cialla et al. shows that TERS can also be successfully used for rapid identification and characterisation of nanoscopic pathogens such as viruses, which is beyond the remit of confocal Raman spectroscopy due to the diffraction limit. However, the exploitation of the full potential of TERS for studies of biological samples in general and pathogens in particular, requires development of more active TERS probes with high plasmonic enhancement that can capture dynamics on a cell surface with a low spectra integration time. The more active probes can also preclude thermal damage to the samples by using low laser power and permit high-resolution chemical imaging instead of only point spectroscopic measurements. A persistent problem in performing TERS on biological samples is the contamination of TERS probes with the sample molecules, which often leads to spurious results. Low-force AFM mode such as non-contact AFM- 
TERS mode causes less sample damage and therefore is beneficial to study soft biological samples $[17,18,20,21,42,43,50]$.

\section{Lipid membranes}

Supported lipid bilayer and human cells were investigated by Böhme et al. using TERS [18]. To determine the specific components on the cell surface, Palmitoyloleoylphosphatidylcholine/dioleoylphosphatidylserine (POPC/DOPS) - supported bilayer was used as a model cell membrane and its TERS spectra were correlated with the nearfield spectra from human dermal derived keratinocyte $(\mathrm{HaCaT})$ cells. A high signal to noise ratio (SNR) with a reasonably short acquisition time of $1 \mathrm{~s}$ was obtained in all near-field spectra. Characteristic Raman modes of the phosphate-containing head-group, which was expected to be in the vicinity of the TERS tip, could be clearly observed in the near-field spectrum of the lipid bilayer. Several lipid bands were observed in the human cell spectrum along with bands from other cellular components such as oligosaccharides and proteins, thus providing a detailed label-free image of a complex biological sample.

Opilik et al. carried out the nanoscale chemical imaging of phase-separated lipid domains [51]. Using Raman bands from $\mathrm{C}-\mathrm{H}$ stretching vibrations, TERS maps (128 $\times$ 128 pixels) of 1,2-dipalmitoyl (d62)-sn-glycero-3-phosphocholine/1,2-Dioleoyl-sn-glycero-3-phosphocholine (d62-DPPC/DOPC) mixed monolayer on template-stripped (TS) gold surface with $<50 \mathrm{~nm}$ spatial resolution were obtained. These results clearly demonstrated that TERS could be used to obtain reproducible Raman spectra from a very small number of lipid molecules and hence could be used for the nanoscale investigation of cell membranes.

Lipids are the fundamental building blocks of biological cell membranes and mediate the interaction of a cell with its external environment such as drugs, pathogens and neighbouring cells involved in biological processes such as cell signalling. Unlike the labelling fluorescence microscopy techniques, TERS provides label-free measurement of molecular distribution on a cell membrane at the nanoscale. Other advanced Raman techniques such as coherent anti-Stokes Raman spectroscopy (CARS) and stimulated Raman scattering (SRS) are not sensitive enough for the same measurements. Furthermore, the short sampling time of TERS measurements allows insights into the molecular dynamics on the cell membranes, which can be exploited for the rational design of effective drugs. However, to truly achieve this goal in situ TERS measurements on live cell membranes in aqueous environment are desired along with the underpinning metrology to carry out successful TERS imaging in liquid.

\section{Nucleic acids}

TERS investigation of a single RNA strand was carried out by E. Bailo and V. Deckert [43]. Seven TERS spectra were reported along a single cytosine strand with good SNR of about 200:1, originating from 30-60 bases. This indicated that each base contributed an SNR of 3-7 to the near-field spectrum, indicating the single-base sensitivity of the TERS measurements. On the basis of this SNR for a single RNA base, a direct and non-destructive sequencing procedure for deoxyribonucleic acid (DNA) using TERS was proposed.

Molecular characterisation of DNA double strand breaks (DSBs) with TERS was carried out by Lipiec et al. [19]. They investigated the susceptibility of DNA bonds to 
radiation damage by irradiating pUC18 circular plasmid DNA as a thin-film in an aqueous solution using a lamp emitting ultraviolet $\mathrm{C}$ (UVC) radiation. Based on the observation of intense bands associated with $\mathrm{CH}_{2} / \mathrm{CH}_{3}$ and $\mathrm{P}-\mathrm{O}-\mathrm{H}$ bending modes, O-C cleavage was found to be the most common form of DNA damage. From the TERS spectra it could be confirmed that the DSBs mainly resulted from 3'- and 5'- bonds of deoxyribose upon UVC radiation exposure.

Combed double-stranded (DS) DNA immobilised on a glass surface were investigated using TERS by Najjar et al. with a resolution $\approx 9 \mathrm{~nm}$ [8] (Fig. 6). A TERS spectrum with Raman modes of DNA nucleobases and backbone containing phosphodiester and sugar groups was obtained. Single-stranded (SS) and DS DNA were clearly distinguished using O-P-O group modes, which demonstrated that DNA hybridisation could be detected using TERS.

DNA bases have characteristic Raman spectra, and with substantial increase in spatial resolution and sensitivity TERS can be used to identify individual bases of DNA. This can be particularly useful when the amount of sample is not enough for other existing methods as for example forensic and archaeological investigations. However, a routine utilisation of TERS in these cutting edge applications demands significant improvements in the current state of the art of TERS systems.

\section{Amino acids}

The capability of TERS to provide information about the coordination sites of a peptide on a metal surface was demonstrated by Deckert-Gaudig et al. by investigating a short peptide immobilised on a $15 \mathrm{~nm}$ thin single transparent gold nanoplate [20]. They
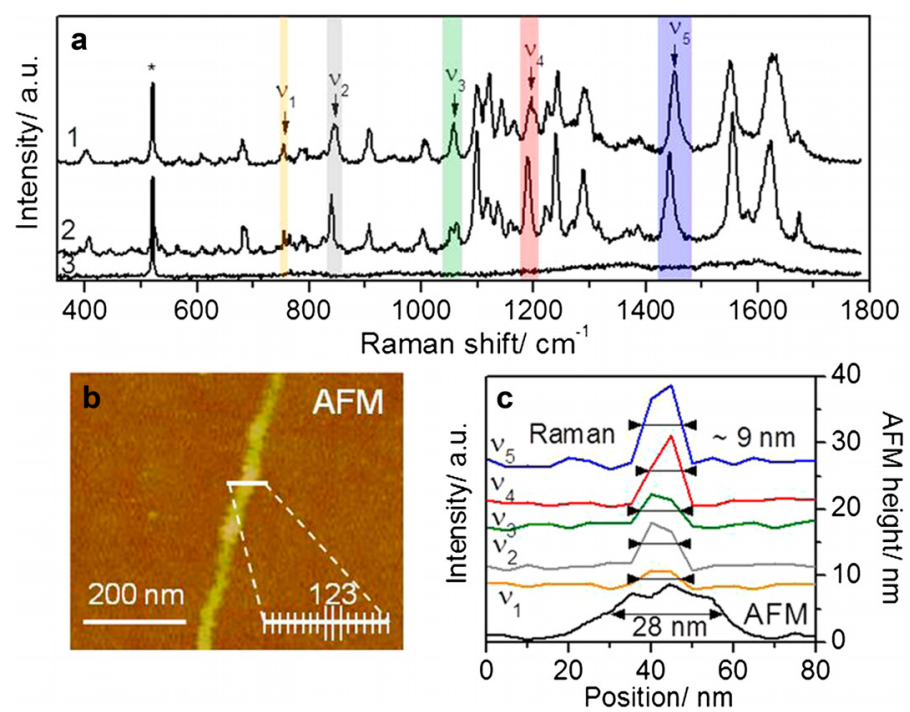

Fig. 6 Investigation of combed double-stranded (DS) DNA immobilised on a glass surface using TERS. a Near-field spectra measured at three different positions, marked as 1, 2, and 3, along the line across the DS DNA bundle in $\mathbf{b}$. The star highlights the characteristic Raman band of Si at $520 \mathrm{~cm}^{-1}$ from the AFM tip. Spectrum integration time: 10 s. b AFM height image of a combed DS DNA bundle. $\mathbf{c}$ Intensity profiles obtained from the integration of near-field bands marked in $\mathbf{a}$ and AFM topography along the line in $\mathbf{b}$. The values of full width at half maximum (FWHM) of the intensity profile of band $\gamma 5$ and AFM topography are indicated. Reprinted with permission from reference [8]. Copyright 2014 American Chemical Society 
determined the structure of oxidized cysteinyl glutathione (CSSG) on the Au surface by analysing the Raman bands corresponding to the glutamic acid, glycine and cysteine units.

To compare the Raman spectra obtained by conventional Raman, SERS and TERS, on a biological sample, Blum et al. carried out a study of bovine serum albumin (BSA), phenylalanine and tyrosine, using all three techniques with the same excitation wavelength [52]. Interestingly, fewer Raman bands were observed in the TERS spectra compared to the SERS and Raman spectra. In contrast to the previous reports, spectral positions of the Raman bands were found to be unchanged in all the three techniques, although the relative band intensities did vary. The results of this study provided useful guidelines for the interpretation of TERS spectra of biological samples and assignment of marker bands for proteins to track their distribution in complex biological specimens.

Kurouski et al. carried out TERS investigation of insulin protofilaments and fibril polymorphs [21]. TERS spectra showed that the twisted insulin fibrils contained a higher concentration of tryosine (Tyr), proline (Pro) and histidine (His) amino acid residues on their surface as compared to flat fibrils, which had a relatively higher surface abundance of cysteine (Cys). Insulin protofilament surfaces were found to have the Raman vibrational mode of carboxyl groups located at twice the frequency compared to the mature fibrils and a very small amount of Tyr and phenylalanine (Phe) amino acid residues compared to both flat and the twisted fibrils. Frequency of the Cys vibrational modes was found to be the same as flat fibrils but twice that of twisted fibrils, indicating that Cys is involved in the formation of twisted fibrils and not the flat insulin fibrils. These results demonstrated the plausible use of TERS to characterise surface amino acid composition and protein secondary structure to understand the mechanisms of fibril growth and propagation that hold the keys to prevent or slow down the progression of various neurodegenerative diseases.

These results indicate that the high spatial resolution and sensitivity of TERS can be exploited to investigate protein structures for pharmaceutical research. Furthermore, inherent complexity of investigating individual molecules calls for underpinning metrology to accurately analyse and interpret TERS spectra. Blum et al. have taken the first step in this direction by comparing Raman spectra of molecules obtained by conventional Raman, SERS and TERS. However, further systematic metrology studies need to be conducted to correlate the Raman peak intensities to the actual amount of substance being probed. In this regard, establishment of a database containing the standardised TERS spectra would be highly beneficial.

\section{Catalysis}

Properties of catalytically-active surfaces are known to be spatially and temporally heterogeneous [53]. Hence, rational design of catalysts requires identification of catalyticallyactive sites and measurement of their local properties. Due to its nanoscale spatial resolution, TERS has the potential to monitor chemical reactions and molecular dynamics occurring at a single catalytic site. Lantman et al. demonstrated this with a photocatalytic reaction in which an Ag-coated TERS tip acted both as a catalytic site as well as a nanoscale antenna to plasmonically enhance the Raman signal from the analytes [10]. On exposure to a green laser $(532 \mathrm{~nm})$, a self-assembled monolayer (SAM) of p-nitrothiophenol (pNTP) molecules adsorbed on Au nanoplates underwent a 
photocatalytic reduction at the apex of TERS tip to p,p'-dimercaptoazobisbenzene (DMAB). The reaction was monitored with TERS spectra collected using a red laser $(633 \mathrm{~nm})$. Time series Raman spectra before and after the reaction clearly showed the Raman bands corresponding to pNTP decrease and DMAB increase in intensity over time.

Recently, Kumar et al. have reported nanoscale chemical mapping of a photocatalytic reaction using TERS [54]. In this study photocatalytic oxidation of $p$-mercaptoaniline (pMA) to $p, p^{\prime}$-dimercaptoazobenzene (DMAB) on a silver catalyst surface was used as a model reaction. The authors first mapped the reaction at a single point of contact of the Ag-coated TERS tip with a reactant substrate. The reaction was then inhibited by protecting the TERS tip with a thin layer of alumina while maintaining the near-field enhancement of the probe. These alumina-protected probes were then used to map the reaction-spots on an Ag-substrate containing a distribution of Ag nanoparticles with $20 \mathrm{~nm}$ spatial resolution as shown in Fig. 7.

Despite the known advantages of a high chemical sensitivity and spatial resolution, only a handful of TERS studies of catalytic reactions have been reported so far [10,54,55]. However, the recent work by Kumar et al. demonstrates that it is possible to image the reaction at a catalytically-active site with nanoscale resolution. The use of alumina-protected TERS probes is an effective method of blocking the catalytic interference from $\mathrm{Ag}$ and $\mathrm{Au}$ while maintaining their enhancement factor. This paves the way for the use of TERS for investigation of heterogeneous catalytic reactions on solid-liquid and solid-gas interfaces.

\section{Polymer blends}

TERS has been successfully applied to the study of polymer-blends revealing numerous novel insights. Nanoscale probing of the surface composition of a polystyrene-polyisoprene (PS-PI) blend film with AFM-TERS was carried out by Yeo et al. [56]. In this study, TERS
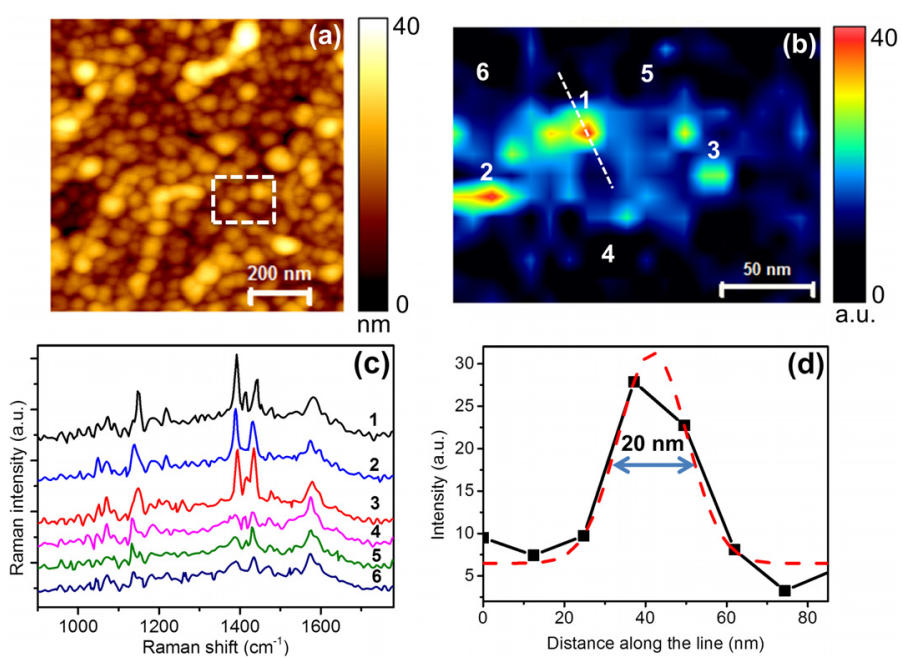

Fig. 7 Mapping of a catalytic reaction at the nanoscale using TERS. a AFM height image showing the distribution of Ag nanoparticles on a glass substrate. $\mathbf{b}$ TERS map made using the $1142 \mathrm{~cm}^{-1}$ peak of DMAB from the dotted rectangle marked in (a) indicates the locations where the catalytic reaction takes place. $\mathbf{c}$ Near-field spectra from the locations marked in (b) shows the characteristic Raman peaks of the reaction product, DMAB at locations 1, 2 and 3, whereas Raman peaks of only the reactant, pMA, are seen in the spectra from locations 5, 6 and 7. Integration time: $0.5 \mathrm{~s}$. d Intensity profile along the line marked in (b) along with the fitted Gaussian curve shows the $20 \mathrm{~nm}$ spatial resolution of the TERS map. Reproduced from [54] with permission from The Royal Society of Chemistry 
was used to study polymers with low Raman scattering cross sections for the first time. The authors identified PI and PS at the surface and sub-surface, respectively and discovered the presence of sub-surface nanopores in the PS film; these results couldn't be obtained using other characterisation techniques. However, only individual TERS spectra from different locations on the surface of the polymer blend were obtained in this work.

Xue et al. reported the first high-resolution TERS image of a poly (methyl methacrylate) (PMMA):poly (styrene-co-acrylonitrile) (SAN) polymer blend [57]. A contrast of 15 was achieved in the near-field vs far-field spectra that allowed high resolution mapping of the blend with a much smaller exposure time. Phase-separation behaviour of the blend was monitored using TERS mapping after 2 and 5 min of annealing at $250{ }^{\circ} \mathrm{C}$. A detailed analysis of the interface of different phases showed an interfacial region of $200 \mathrm{~nm}$ at an early stage of phase evolution. A phase inversion due to an unexpected transition of PMMA from the dispersed phase to the continuous phase was observed.

The studies discussed in this section indicate that the interfaces between the polymer blend components can be successfully investigated with a nanoscale resolution using TERS. However, thermal degradation of some polymer films may contaminate a TERS probe, hence, a low laser power on the sample may be required to acquire a TERS spectrum. A combination of low laser power and low Raman cross-section of polymerblends increases the integration time for spectra with SNR > 10, and can lead to TERS imaging times of several hours putting stringent demands on the stability of the TERS system [57]. These issues can be overcome by development of TERS probes with high plasmonic enhancement providing a high SNR in the near-field spectra with a relatively short integration time.

\section{Organic solar-cells}

The photophysical processes in the photovoltaic solar-cell polymer blends occur at the nanoscale [58]. The morphology of the blend components determines the efficiency of exciton dissociation and charge transport to the electrodes. Therefore, optimisation of blend morphology is the major bottleneck in increasing the power conversion efficiency of solar-cell devices. TERS has been successfully utilised for nanoscale chemical mapping of the components in the photovoltaic polymer blends. Wang et al. demonstrated this by TERS imaging of domains in poly (3-hexylthiophene):[6, 6]penyl-C61 butyric acid methyl ester (P3HT:PCBM) films for solar-cells at optical resolution of $9 \mathrm{~nm}$ [9]. They investigated the influence of thermal annealing $\left(150{ }^{\circ} \mathrm{C}\right.$ for 5 and $30 \mathrm{~min}$ ) on photovoltaic blend films and found that the PCBM molecules aggregated into micrometre- and nanometre-size clusters after annealing times of 30 and $5 \mathrm{~min}$, respectively. By acquiring the morphological and spectroscopic information simultaneously, the authors were able to quantitatively reveal the local photoluminescence (PL) quenching efficiency, which is related to the electron transfer from P3HT to PCBM.

Many solar-cell polymer blends rapidly degrade in ambient air due to presence of oxygen and moisture under laser illumination $[59,60]$. Therefore, the TERS measurements require an inert environment such as nitrogen or argon. Furthermore, in TERS spectra from these samples a broad PL band such as that from P3HT may overlap with the Raman bands; therefore, a reliable fitting procedure is required to accurately deconvolute the Raman and PL bands in order to measure their intensities [61]. 


\section{Crystalline and semiconductor materials}

The non-destructive and high-throughput requirements of the semiconductor industry make Raman spectroscopy the technique of choice for the characterisation of semiconductor materials [62]. With the shrinking of semiconductor devices to the nanoscale, TERS is expected to play a significant role in their chemical characterisation. Silicon is the first and the most investigated bulk semiconductor using TERS so far [11, 63-72]. Because the spatial fluctuations of strain can be deleterious for the performance of silicon devices, the nanoscale probing of strain in silicon has stimulated significant interest [63-65, 69-71]. Introduction of strain in silicon using a SiGe buffer layer is a wellknown technique to enhance carrier mobility [73], which was used by Intel for the first time in the $90 \mathrm{~nm}$ process technology [74]. The strain-induced shift of the first order $520 \mathrm{~cm}^{-1}$ Raman peak associated with optical phonons in silicon has been used to spatially map stress variations using TERS, with a lateral resolution of 20-25 nm [11, 70]. A similar resolution has been achieved in the strain-mapping of patterned SiGe structures [75].

A practical issue in the TERS measurements on bulk semiconductors is the presence of far-field artefacts in the near-field signal. For silicon, this has been addressed in several works by means of crystal symmetry considerations [11, 63, 65-69]. This idea exploits the fact that the TERS tip induces a depolarisation in the near-field, thus allowing separation of the far-field signal contribution (polarised) from the near-field signal (depolarised) using an analyser. With this approach, a maximum contrast of 40 has been achieved [66]. TERS on SiC samples has been reported by K. F. Domke and B. Pettinger, who used graphene adlayers conductivity to keep an STM tip into tunnelling contact with the surface [76]. This work also highlighted the signal enhancement of different Raman vibrational modes due to presence of electrical charge on the sample surface.

The ability of TERS to probe the phonon symmetry at the nanoscale has been demonstrated by studying $\mathrm{LiNbO}_{3}$ [77] and $\mathrm{BaTiO}_{3}$ [78] nanocrystals. In particular, the longitudinal optical (LO) mode of $\mathrm{BaTiO}_{3}$, which is characteristic of the tetragonal phase and consequently of the ferroelectric state, has been used to image ferroelectric domains as shown in Fig. 8 [78]. This study provided evidence of spontaneous
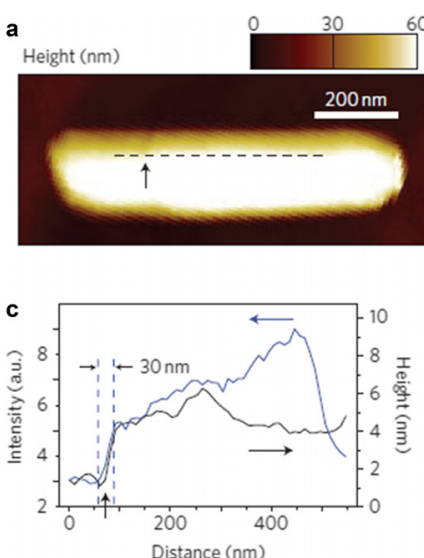

b

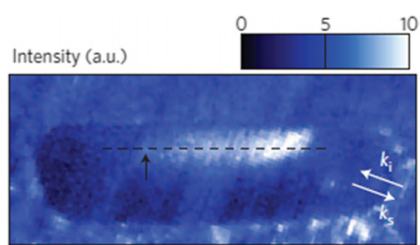

d

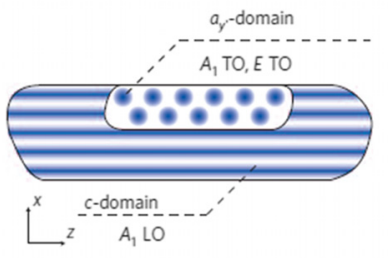

Fig. 8 Optical nanocrystallography using spatially-resolved TERS. a Topography image of a BaTiO $\mathrm{B}_{3}$ nanorod. b Ferroelectric domain image acquired using the spectrally integrated TERS signal. c Cross-section profiles along the dashed lines marked in (a) and (b). d Assignment of domains according to the Raman selection rules for the TERS configuration used in reference [78]. Reprinted by permission from Macmillan Publishers Ltd: NATURE NANOTECHNOLOGY [78], copyright (2009) 
ferroelectric domains within a single crystal of $\mathrm{BaTiO}_{3}$ with a size of several hundreds of nanometres, comparable with the usual size of single domains in bulk dielectrics [78].

A few TERS studies have been reported on III-V nitride semiconductors $[79,80]$. The relative intensities of the Raman modes have been used to probe orientations of the crystal faces of GaN thin-films at the nanoscale [79]. Nanoscale defects have been identified by means of the forbidden GaN Raman mode at $668 \mathrm{~cm}^{-1}$, which is otherwise undetectable using conventional Raman spectroscopy due to its weak intensity [79]. Vertically-aligned GaN nanorod arrays with nonpolar InGaN/GaN multi quantum wells (MQW) have been recently investigated using TERS [80]. Fluctuations of a few percent of In content in the InGaN layer were identified with a lateral resolution below $35 \mathrm{~nm}$ along with the chemical composition, charge distribution and strain in the MQW [80].

TERS brings several advantages in terms of non-destructiveness and the variety of information that can be garnered at the nanoscale from the crystalline and semiconductor materials. In contrast to confocal Raman spectroscopy, the assumption of Raman scattering being an incoherent process is not valid for TERS, and this could be used to measure the correlation length of phonons in crystals (both in their bulk and 2-D forms), which has so far been obtained for graphene only [81]. The polarisation of the incident and scattered field is also valuable for the study of crystalline materials as it is directly linked to their crystalline arrangement which, in turn, determines their symmetry and vibrational properties. However, a major challenge for TERS is that the near-field polarisation is dictated by the plasmon excitation as well as by the morphology of the tip, the latter being less reproducible.

\section{1-D materials}

Carbon nanotubes (CNTs) are the most widely-studied nanomaterials using TERS so far [12, 46, 82-100]. 1-D structures like single wall CNTs (SWCNTs) are the preferred samples to demonstrate the high resolution capability of a TERS instrument. These have also been used to calculate contrast and enhancement factor of TERS probes. A novel methodology to estimate the enhancement factor of a TERS probe using a CNT was proposed by Roy et al. [101]. This method involved deconvoluting the near-field and far-field Raman signals by fitting two Gaussian curves to the line profile across the TERS image of a SWCNT [101]. Roy and Williams demonstrated that a radially polarised annular beam improves both the spatial resolution and the contrast of a TERS measurement [92]. Weber-bargioni et al. used SWCNTs to test the spatial resolution of novel nanofabricated coaxial antenna tips [97]. More recently, Chen et al. used CNTs to demonstrate a spatial resolution of $1.7 \mathrm{~nm}$ with an STM-TERS system at roomtemperature [46].

The synthesis of CNTs results in bundles that often contain a variety of tubes with different structural properties. Since the first TERS experiments, near-field Raman spectroscopy has been a versatile technique to probe nanoscale variations in chirality, diameter, defects, strain and doping of CNTs $[82,83]$. Anderson et al. performed TERS imaging on arc-discharge and CVD-grown SWCNTs [84] and found a high localisation of the radial breathing mode (RBM) intensity in the arc-discharge SWCNTs, compared to the CVD-grown SWCNTs, which was attributed to the spatial variations in structure along the tubes. Georgi and Hartschuh estimated the spatial extent $(\approx 2 \mathrm{~nm})$ of the 
scattering process responsible for the D-peak (defect-activated peak in carbon systems) [91]. Because of the strong coupling of the electrons and phonons in $\mathrm{sp}^{2}$-hybridised carbon structures, doping can be easily probed using Raman spectroscopy [102]. Maciel et al. investigated nitrogen-, phosphorous- and boron-doped SWCNTs using TERS, and found evidence of a defect-activated peak, that was called $G_{D}^{\prime}\left(G^{\prime}\right.$ mode at $\sim 2700 \mathrm{~cm}^{-1}$ in defective graphite) [87]. Using the frequency of the $G_{D}^{\prime}$ mode they were able to localise the electron and phonon confinement near the defect sites, and discriminated between $\mathrm{n}$ - and p-doping. In the same experimental set-up, near-field photoluminescence showed a highly localised light emission from the negatively charged defects. Peica et al. demonstrated the capability of TERS to differentiate the CNT diameters by means of the nanoscale observation of the RBMs [93]. Chirality of the tubes was determined using a combination of RBM and $\mathrm{G}^{-}$mode frequencies [93]. Similarly, chirality variations within a single CNT have been reported by Anderson et al. [12, 86]. Recently, Okuno et al. were able to use TERS to identify the localised deformations at the position where two CNTs cross each other and observed a transition from semiconducting to metallic behaviour, indicated by the asymmetric contribution to the shape of the $\mathrm{G}^{-}$-band (Fig. 9) [12]. Similarly, Yano et al. studied the effect of strain on near-field Raman scattering by intentionally deforming the CNTs with an AFM tip [100]. The level of strain was
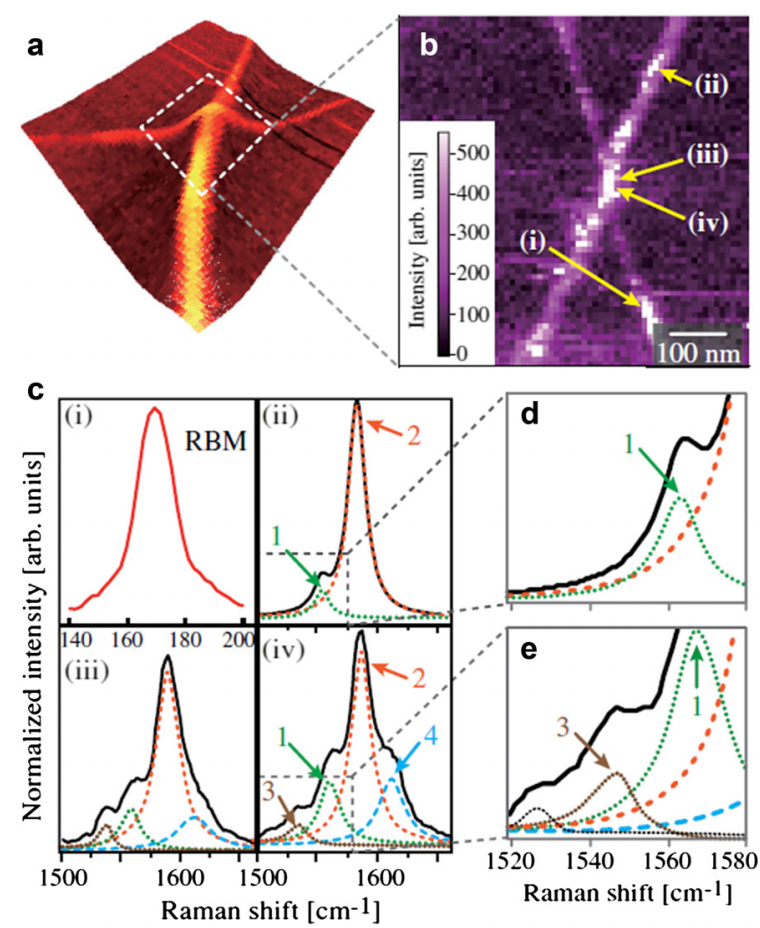

Fig. 9 TERS investigation of semiconductor to metal transition of CNT. a 3-D AFM image of two carbon nanotubes crossing each other. $\mathbf{b}$ TERS map of the $\mathrm{G}^{+}$- band intensity, collected in the area marked with the white dashed line in (a). c TERS spectra from points (i)-(iv) at the positions marked in (b). Spectrum at point (i) shows the frequency region where the RBM peak is present. Spectrum at point (ii) shows the G-band, composed of two Lorentzian peaks, corresponding to the $\mathrm{G}^{-}$- mode (peak 1) and the $\mathrm{G}^{+}$- mode (peak 2). Spectrum at point (iii) and (iv) shows two additional peaks, with asymmetric Fano (peak 3) and Lorentzian (peak 4) line-shape, respectively. $\mathbf{d}$ Magnification of the spectra far (point ii) and near (point iv) the junction. Reprinted with permission from reference [12], Copyright 2013 by the American Physical Society 
found to depend on the chirality and the diameter of the CNTs, which was attributed to the variation in friction coefficients of different CNTs [100].

TERS has also been applied to study nanotubes and nanowires other than CNTs. Marquestaut et al. studied isolated GaN nanowires, and reported that the near-field enhancement factor depends on the symmetry of the specific vibrational mode [103]. Böhmler et al. were able to image single CdSe nanowires using tip-enhanced Raman and PL spectroscopy, thus differentiating the PL energy emission of nanowires with different diameters [104]. Finally, Ogawa et al. reported a nanoscale TERS mapping of a single Ge nanowire, and estimated the fraction of crystalline and amorphous content of Ge [105]. These studies demonstrate the progress in nanoscale spectroscopy and imaging of 1-D nanostructures using TERS in order to obtain new information about these materials.

As the aforementioned studies indicate, 1-D nanostructures such as SWCNTs are convenient samples to test the spatial resolution of a TERS system due to their small radius, which is $<1 \mathrm{~nm}$. Therefore, SWCNTs are good candidate as a metrological reference sample to compare the spatial resolution of TERS systems in different research labs.

\section{2-D materials}

The discovery of graphene in 2004 has given rise to a surge in research activities on 2D materials with novel properties, which can be used in applications such as electronics, optoelectronics, spintronics, batteries and catalysis, and can be stacked in heterostructures to engineer new materials [106]. Confocal Raman spectroscopy has been a primary tool to characterise 2-D materials. This is especially valid for graphene, which shows two prominent features in the Raman spectrum, due to in-plane vibrations of the carbon atoms: the G-peak at $1580 \mathrm{~cm}^{-1}$ and the 2 D-peak at $2700 \mathrm{~cm}^{-1}$ [102]. The D-peak is observed at $1350 \mathrm{~cm}^{-1}$, which is activated in the presence of defects [102]. 2D-peak is the second order overtone of the D-peak. The high sensitivity and spatial resolution of TERS can be a powerful tool particularly for nanoscale 2-D devices [107]. The first reported TERS spectrum of graphene by Hoffmann et al. dates back to 2008 [108]. In the following year, Domke and Pettinger reported a study on 6H-SiC substrates with graphene adlayers using STM-TERS [76]. The TERS signal enhancement was found to be lower for graphene than $\mathrm{SiC}$, and this discrepancy was attributed to polarisation-dependence of the enhancement. In the same year, Saito et al. published a TERS map of a few-layers graphene flake in transmission mode, with a spatial resolution of $\approx 30 \mathrm{~nm}$. Fluctuations in the 2D-peak within a single flake were attributed to a local strain distribution, whereas the local variation in the G-peak width and position, suggested an inhomogeneous and unintentional doping. A theoretical treatment of the near-field enhancement in 2-D systems has been recently published [109], although experimental verification is yet to be reported. Despite the high spatial resolution of TERS maps, it is observed that enhancement of the Raman bands of graphene is weak due to their in-plane vibrations [76]. The near-field interactions between the probe and graphene are complex due to orientations of the electric field and the in-plane Raman tensor. Hence, the mechanism of signal enhancement in graphene is yet to be fully understood. Stadler et al., performed TERS mapping of CVD-grown and mechanically-exfoliated single-layer graphene with a resolution of less than $12 \mathrm{~nm}$ [110]. They were able to identify defects, edges, hydrogen-terminated and other 

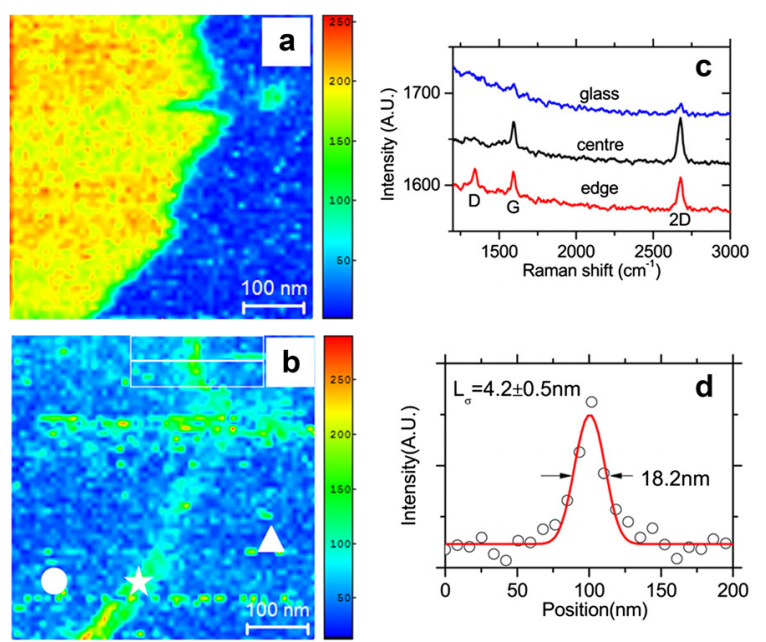

Fig. 10 Visualising graphene edge using TERS. a AFM lateral force image and (b) TERS map of the D-peak intensity of a single-layer graphene flake. c TER spectra corresponding to the points marked with circle (centre of the flake), star (edge of the flake) and triangle (glass substrate) in (b). $\mathbf{d}$ (open circles) TERS D-peak intensity profile at the edge of the flake, averaged in the rectangular area marked in (b), (solid line) calculated D-peak intensity spatial profile showing spatial width of $18.2 \mathrm{~nm}$. Reprinted with permission from reference [13], Copyright 2013, American Vacuum Society

contaminated areas within a graphene flake with sub-diffraction-limited resolution, which otherwise cannot be resolved with confocal Raman spectroscopy.

Several works on graphene have focused on the Raman characterisation of defects, using the D-peak. These include point defects, i.e. vacancy or dopant, or line defects, i.e. grain boundaries. Despite the reported TERS resolution of $\approx 1 \mathrm{~nm}$ for singlemolecule imaging in vacuum (see the next section for details), mapping of a single nanometre-sized defect on graphene using TERS has not been reported yet. On the other hand, $\mathrm{Su}$ et al. reported TERS image of a graphene edge using the D-peak intensity. The spatial extent of the D-peak, i.e. the phase-breaking length was evaluated to be $\approx 4.2 \mathrm{~nm}$ [13], which is of the same order of magnitude as reported in the literature [111] (Fig. 10).

Structural deformations in 2-D materials can also be induced in-situ by means of the TERS tip. For instance, Snitka et al. fabricated tips by flattening Au microwires for tapping mode AFM-TERS, and studied the effect of tip pressure on graphene [112]. They observed that the tip-induced local strain caused fluctuations in the 2D-peak intensity. Analogously, Weng et al. reported TERS experiments where a suspended graphene sheet was locally deformed by applying a voltage to the tip. Using this approach they estimated the Young's modulus of graphene to be $1.48 \mathrm{TPa}$ [113].

Currently, the scientific research on applications of 2-D materials in nanoscale optoelectronic devices is quite fervent around the world. Hence, evaluation of the quality, defect density and localised properties of devices based on 2-D materials necessitates the need for nanoscale characterisation. A tip-enhanced optical apparatus can not only be used for high resolution Raman mapping of such devices but also can also allow simultaneous multi-parameter measurements of photocurrent, PL and second harmonic generation. Therefore, a tip-enhanced setup may be regarded as a broad nanoscale optical spectroscopy tool, which can be applied to the study of localised PL and excitonic 
properties in 2-D transition metal dichalcogenides. However, the effect of doping from the metal-coating of the tip is likely to influence such measurements. Hence, protection of the TERS probes with an ultrathin insulating layer such as alumina can minimise or block such tip-sample interaction while still maintaining the plasmonic enhancement at the tip-apex allowing accurate measurement of the intrinsic local vibrational and excitonic properties of such 2-D materials.

\section{Single molecule detection}

Single molecule spectroscopy has fascinated scientists for many decades and attempts have been made to understand the difference in molecular behaviour in an ensemble [114]. Even though single-molecule imaging with atomic resolution is achievable using non-contact AFM [115] and STM [116], chemical information cannot be obtained with these techniques. The first demonstration of single-molecule detection with Raman spectroscopy dates back to 1997 - that was accomplished using SERS enhancement from metal nanoparticles on single rhodamine $6 \mathrm{G}$ molecules at room temperature [117]. Raman polarisation behaviour and sudden temporal spectral changes supported the evidence that Raman signal was being detected from a single molecule [117]. About a decade later single molecule sensitivity on a length scale of $10 \mathrm{~nm}$ was reported using TERS $[118,119]$. Using AFM-TERS, Neacsu et al. probed single molecules of malachite green adsorbed on a metal surface [118]. The detection of an individual molecule was supported by the observation of temporal variations in the relative intensities of the peaks. Similar observations were reported by Zhang et al. using STM-TERS to investigate brilliant cresyl blue (BCB) on a metal substrate [119]. The temporal fluctuations of the intensities of the Raman peaks were used to support the detection of a single or a few molecules. In 2006, Steidtner and Pettinger imaged BCB molecules using ultra-high vacuum (UHV) TERS, which decreases photobleaching, thus allowing much longer laser exposure times than in the presence of oxygen [120]. The sharp focus $(\approx \lambda / 2)$ obtained by a parabolic mirror with high NA along with an enhancement of $10^{6}$ due to the gap-mode

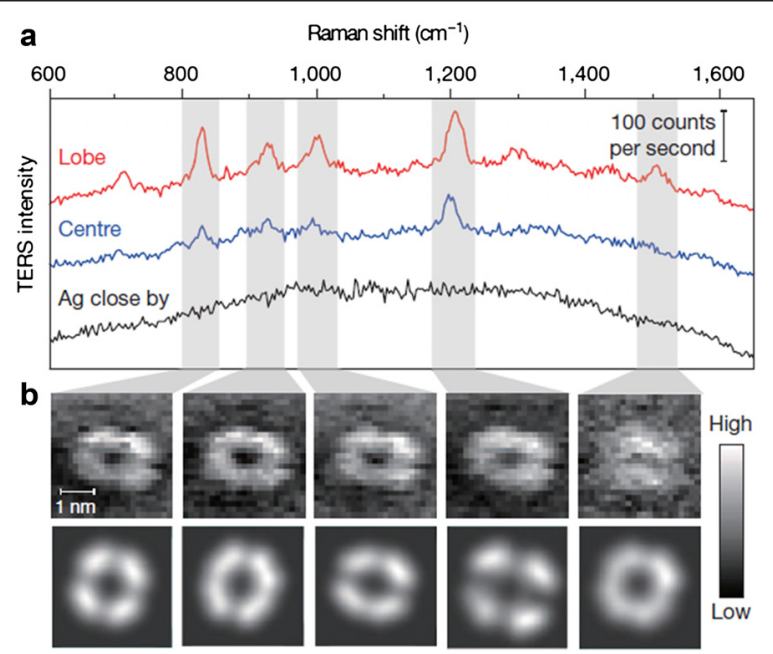

Fig. 11 Chemical mapping of a single molecule using TERS. a TER spectra of a lobe and the centre of an individual $\mathrm{H}_{2}$ TBPP molecule and the silver substrate (b) TERS maps of different Raman modes from an individual $\mathrm{H}_{2}$ TBPP molecule (top panel) and the corresponding TERS maps simulated with density functional theory (DFT). Reprinted with permission from Macmillan Publishers Ltd: NATURE [7], copyright 2012 
configuration, made the mapping of a single molecule possible with a spatial resolution of $15 \mathrm{~nm}$ [121]. More recently, the imaging of a single molecule of $\mathrm{H}_{2}$ TBPP with sub-nm resolution (Fig. 11) has been achieved, with a clear resolution of the four distinct lobes of the molecule [7]. This record resolution was obtained by means of a finely-tuned doubleresonance mechanism responsible for a large field enhancement. As reported previously in similar studies, the laser wavelength was chosen to match a vibronic transition of the molecule $[118,119,121]$. Additionally, the gap between the metal tip and the metal substrate, was tuned to match the nanocavity plasmons with the energy of the Raman scattered photons.

These single molecule studies demonstrate the sensitivity and spatial resolution achievable in TERS experiments. The capability to map molecules at the nanoscale will in future provide powerful new insights into molecular adsorption and reaction dynamics at surfaces, ultimately enabling manipulation of process selectivity, for example in catalytic reactions.

\section{Review and conclusion}

In the last 15 years, TERS has emerged to be an effective tool for nanoscale chemical and structural characterisation of surfaces. Being an ambient technique it is rapidly defining its unique capability as a label-free nondestructive nano-tool. TERS doesn't require high vacuum or cryogenic temperatures for its operation and has been applied in both air and liquid environments. At the early stage, nanoscale materials with large Raman cross-sections such as carbon nanotubes have been the preferred sample; however, recently biological and crystalline materials having much smaller Raman crosssections, have also been characterised at the nanoscale. The investigation of biological samples such as pathogens, lipid membranes, nucleic acids and amino acids highlight the possibility of chemical measurements of biological system at the nanometre lengthscale without labelling. Another area where TERS is likely to have a large impact is the field of nanoscale electronic and optoelectronic devices employing semiconductor, 1-D and 2-D materials. As the device size shrinks below $20 \mathrm{~nm}$, TERS would find a niche in characterising these devices.

For a long time the issues related to tip-yield and reproducibility have slowed down the utilisation of TERS. However, significant progress has been made to resolve these problems with several groups reporting nearly $100 \%$ yield of TERS probes [35, 36]. The strategy of reducing the refractive index of the AFM tip prior to metal deposition has proved to be very effective in improving the plasmon resonance characteristics of the TERS probes. Furthermore, a recent interlaboratory study carried out by Zenobi group has demonstrated that TERS spectra from the same sample can be reproduced in different labs around the world [6]. Furthermore, reference samples are required for verifying the performance of TERS instruments, TERS probes and optimisation of tipfabrication procedures. Progress has been made in this direction and effective reference methodologies for an accurate characterisation and comparison of TERS probes have been reported $[29,101,122]$. These reference methods are expected to accelerate uptake of the technique and facilitate development of commercial TERS probes.

The physics of high signal-enhancement mechanism and sub-nm resolution is not fully understood yet. Recent results of $0.5 \mathrm{~nm}$ spatial resolution and imaging a single molecule by Zhang et al. have opened up the debate on the singular contribution of the electric field enhancement in the gap-mode [7]. Chemical enhancement is attributed to 
be the mechanism for very high signal enhancement; however, it does not explain the unexpectedly high spatial resolution observed in the recent report [7]. The possibilities of stimulated Raman scattering and contribution of hot-electrons have been invoked to explain the mechanism of high enhancement [123]. Further research is required to understand the enhancement mechanism in TERS. However, with the recent progress in tip-yield, automation of instrumentation, and preparation of effective reference samples and standards, TERS can become a routine analytical technique with unique capability of chemical characterisation at $<50 \mathrm{~nm}$ spatial resolution.

\section{Competing interests}

The authors declare that they have no competing interests.

\section{Authors' contributions}

The authors discussed and prepared an outline of the manuscript. NK contributed to the sections on introduction, TERS principles and instrumentation, application of TERS in biology, catalysis, polymer-blends and Review and conclusion. WS contributed to the sections on TERS principles and instrumentation. SM contributed to the sections on application of TERS in semiconductors, single molecule detection, 1-D and 2-D materials. All sections of the manuscript were critically reviewed and edited by DR. All authors read and approved the final manuscript.

\section{Acknowledgements}

NK, SM, and DR gratefully acknowledge financial support from the Innovation, Research and Development programme of the National Measurement System, UK (Project No: 115948). NK and DR acknowledge financial support from the NEW02 project of the European metrology research programme (EMRP) (Project No: 116326). WS acknowledges financial support from Natural Sciences Foundation of China (Grant No: 61306115 and 61178039) and China Postdoctoral Science Foundation (Grant No: 2013 M541807).

\section{Author details}

${ }^{1}$ National Physical Laboratory, Hampton Road Teddington, Middlesex TW11 OLW, UK. ${ }^{2}$ Institute of Materials Physics, Hangzhou Dianzi University, 310018 Hangzhou, China. ${ }^{3}$ Ningbo Institute of Materials Technology \& Engineering, Chinese Academy of Sciences, 315201 Ningbo, China.

Received: 29 October 2014 Revised: 7 May 2015 Accepted: 22 May 2015

Published online: 01 July 2015

\section{References}

1. Wessel J. Surface-enhanced optical microscopy. J Opt Soc Am B, Opt Phys. 1985;2:1538-41.

2. Stockle RM, Suh YD, Deckert V, Zenobi R. Nanoscale chemical analysis by tip-enhanced Raman spectroscopy. Chem Phys Lett. 2000;318:131-6.

3. Hayazawa N, Inouye Y, Sekkat Z, Kawata S. Metallized Tip amplification of near-field Raman scattering. Opt Commun 2000;183:333-6.

4. Anderson MS. Locally enhanced Raman spectroscopy with an atomic force microscope. Appl Phys Lett. 2000;76:3130-2.

5. Pettinger B, Picardi G, Schuster R, Ertl G. Surface enhanced Raman spectroscopy: towards single molecular spectroscopy. Electrochemistry. 2000;68:942-9.

6. Blum C, Opilik L, Atkin JM, Braun K, Kammer SB, Kravtsov V, et al. Tip-enhanced Raman spectroscopy - an interlaboratory reproducibility and comparison study. J Raman Spectrosc. 2014;45:22-31.

7. Zhang R, Zhang Y, Dong ZC, Jiang S, Zhang C, Chen LG, et al. Chemical mapping of a single molecule by Plasmon-enhanced Raman scattering. Nature. 2013;498:82-6.

8. Najjar S, Talaga D, Schue L, Coffinier Y, Szunerits S, Boukherroub R, et al. Tip-enhanced Raman spectroscopy of combed double-stranded DNA bundles. J Phys Chem C. 2014;118:1174-81.

9. Wang X, Zhang D, Braun K, Egelhaaf HJ, Brabec CJ, Meixner AJ. High-resolution spectroscopic mapping of the chemical contrast from nanometer domains in P3HT:PCBM organic blend films for solar-cell applications. Adv Funct Mater. 2010;20:492-9.

10. van Schrojenstein Lantman EM, Deckert-Gaudig T, Mank AJG, Deckert V, Weckhuysen BM. Catalytic processes monitored at the nanoscale with tip-enhanced Raman spectroscopy. Nat Nanotechnol. 2012;7:583-6.

11. Lee N, Hartschuh RD, Mehtani D, Kisliuk A, Maguire JF, Green M, et al. High contrast scanning nano-Raman spectroscopy of silicon. J Raman Spectrosc. 2007;38:789-96.

12. Okuno Y, Saito Y, Kawata S, Verma P. Tip-enhanced Raman investigation of extremely localized semiconductor-to-metal transition of a carbon nanotube. Phys Rev Lett. 2013;111:216101.

13. Su W, Roy D. Visualizing graphene edges using tip-enhanced Raman spectroscopy. J Vac Sci Technol B. 2013:31:041808.

14. Yeo BS, Stadler J, Schmid T, Zenobi R, Zhang WH. Tip-enhanced Raman spectroscopy - its status, challenges and future directions. Chem Phys Lett. 2009;472:1-13.

15. Schmid T, Yeo BS, Leong G, Stadler J, Zenobi R. Performing tip-enhanced Raman spectroscopy in liquids. J Raman Spectrosc. 2009:40:1392-9.

16. Huang B, Bates M, Zhuang XW. Super-resolution fluorescence microscopy. Annu Rev Biochem. 2009;78:993-1016. 
17. Wood BR, Bailo E, Khiavi MA, Tilley L, Deed S, Deckert-Gaudig T, et al. Tip-enhanced Raman scattering (TERS) from hemozoin crystals within a sectioned erythrocyte. Nano Lett. 2011;11:1868-73.

18. Bohme R, Richter M, Cialla D, Rosch P, Deckert V, Popp J. Towards a specific characterisation of components on a cell surface - combined TERS-investigations of lipids and human cells. J Raman Spectrosc. 2009;40:1452-7.

19. Lipiec E, Sekine R, Bielecki J, Kwiatek WM, Wood BR. Molecular characterization of DNA double strand breaks with Tip-enhanced Raman scattering. Angew Chem Int Ed. 2014;53:169-72.

20. Deckert-Gaudig T, Bailo E, Deckert V. Tip-enhanced Raman scattering (TERS) of oxidised glutathione on an ultraflat gold nanoplate. Phys Chem Chem Phys. 2009;11:7360-2.

21. Kurouski D, Deckert-Gaudig T, Deckert V, Lednev IK. Surface characterization of insulin protofilaments and fibril polymorphs using Tip-enhanced Raman spectroscopy (TERS). Biophys J. 2014;106:263-71.

22. Richards D, Milner RG, Huang F, Festy F. Tip-enhanced Raman microscopy: practicalities and limitations. J Raman Spectrosc. 2003;34:663-7.

23. Hayazawa N, Watanabe H, Saito Y, Kawata S. Towards atomic site-selective sensitivity in tip-enhanced Raman spectroscopy. J Chem Phys. 2006;125:244706.

24. Asghari-Khiavi M, Wood BR, Hojati-Talemi P, Downes A, McNaughton D, Mechler A. Exploring the origin of tip-enhanced Raman scattering; preparation of efficient TERS probes with high yield. J Raman Spectrosc. 2012;43:173-80.

25. Kawata S, Shalaev VM. Tip enhancement. Amsterdam: Elsevier; 2007.

26. Zhang M, Wang R, Zhu Z, Wang J, Tian Q. Experimental research on the spectral response of tips for tip-enhanced raman spectroscopy. J Opt. 2013;15:055006.

27. Park KD, Kim YH, Park JH, Park JS, Lee HS, Yim SY, et al. Ultraviolet tip-enhanced nanoscale Raman imaging. J Raman Spectrosc. 2012;43:1931-4.

28. GarciaVidal FJ, Pendry JB. Collective theory for surface enhanced Raman scattering. Phys Rev Lett. 1996;77:1163-6.

29. Kumar N, Rae A, Roy D. Accurate measurement of enhancement factor in tip-enhanced Raman spectroscopy through elimination of far-field artefacts. Appl Phys Lett. 2014;104:123106.

30. Lerman GM, Levy U. Effect of radial polarization and apodization on spot size under tight focusing conditions. Opt Express. 2008;16:4567-81.

31. Wang J, Wu X, Wang R, Zhang M. Detection of Carbon Nanotubes Using Tip-Enhanced Raman Spectroscopy. In: Marulanda JM, editor. Electronic Properties of Carbon Nanotubes. Vukova: InTech; 2011.

32. Stadler J, Schmid T, Zenobi R. Nanoscale chemical imaging using Top-illumination Tip-enhanced Raman spectroscopy. Nano Lett. 2010;10:4514-20.

33. Wang P, Zhang D, Li L, Li Z, Zhang L, Fang Y. Reversible defect in graphene investigated by Tip-enhanced Raman spectroscopy. Plasmonics. 2012;7:555-61.

34. Zhang D, Heinemeyer U, Stanciu C, Sackrow M, Braun K, Hennemann LE, et al. Nanoscale spectroscopic imaging of organic semiconductor films by plasmon-polariton coupling. Phys Rev Lett. 2010;104:056601.

35. Yeo BS, Schmid T, Zhang W, Zenobi R. Towards rapid nanoscale chemical analysis using tip-enhanced Raman spectroscopy with Ag-coated dielectric tips. Anal Bioanal Chem. 2007;387:2655-62.

36. Hayazawa N, Yano TA, Kawata S. Highly reproducible tip-enhanced Raman scattering using an oxidized and metallized silicon cantilever tip as a tool for everyone. J Raman Spectrosc. 2012;43:1177-82.

37. Johnson TW, Lapin ZJ, Beams R, Lindquist NC, Rodrigo SG, Novotny L, et al. Highly reproducible near-field optical imaging with Sub-20-nm resolution based on template-stripped gold pyramids. ACS Nano. 2012;6:9168-74.

38. Williams C, Roy D. Fabrication of gold tips suitable for tip-enhanced Raman spectroscopy. J Vac Sci Technol B. 2008:26:1761-4.

39. Neacsu CC, Berweger S, Raschke MB. Tip-enhanced Raman imaging and nanospectroscopy: sensitivity, symmetry, and selection rules. Nanobiotechnology. 2007;3:172-96.

40. Lloyd JS, Williams A, Rickman RH, McCowen A, Dunstan PR. Reproducible electrochemical etching of silver probes with a radius of curvature of $20 \mathrm{~nm}$ for tip-enhanced Raman applications. Appl Phys Lett. 2011;99:143108.

41. Yu J, Saito Y, Ichimura T, Kawata S, Verma P. Far-field free tapping-mode tip-enhanced Raman microscopy. Appl Phys Lett. 2013;102:123110.

42. Neugebauer U, Rosch P, Schmitt M, Popp J, Julien C, Rasmussen A, et al. On the way to nanometer-sized information of the bacterial surface by tip-enhanced Raman spectroscopy. ChemPhysChem. 2006;7:1428-30.

43. Bailo E, Deckert V. Tip-enhanced Raman spectroscopy of single RNA strands: towards a novel direct-sequencing method. Angew Chem Int Ed. 2008;47:1658-61.

44. Rickman RH, Dunstan PR. Enhancement of lattice defect signatures in graphene and ultrathin graphite using tip-enhanced Raman spectroscopy. J Raman Spectrosc. 2014;45:15-21.

45. Ghislandi M, Hoffmann GG, Tkalya E, Xue L, De With G. Tip-enhanced Raman spectroscopy and mapping of graphene sheets. Appl Spectrosc Rev. 2012;47:371-81.

46. Chen C, Hayazawa N, Kawata S. A $1.7 \mathrm{~nm}$ resolution chemical analysis of carbon nanotubes by tip-enhanced Raman imaging in the ambient. Nat Commun. 2014;5:3312.

47. Malard LM, Pimenta MA, Dresselhaus G, Dresselhaus MS. Raman spectroscopy in graphene. Phys Rep. 2009:473:51-87.

48. Weckhuysen BM. In-situ spectroscopy of catalysts. CA: American Scientific Publishers Stevenson Ranch; 2004

49. Rae A, Stosch R, Klapetek P, Walker ARH, Roy D. State of the art Raman techniques for biological applications. Methods. 2014;68:338-47.

50. Cialla D, Deckert-Gaudig T, Budich C, Laue M, Moller R, Naumann D, et al. Raman to the limit: tip-enhanced Raman spectroscopic investigations of a single tobacco mosaic virus. J Raman Spectrosc. 2009;40:240-3.

51. Opilik L, Bauer T, Schmid T, Stadler J, Zenobi R. Nanoscale chemical imaging of segregated lipid domains using tip-enhanced Raman spectroscopy. Phys Chem Chem Phys. 2011;13:9978-81.

52. Blum C, Schmid T, Opilik L, Weidmann S, Fagerer SR, Zenobi R. Understanding tip-enhanced Raman spectra of biological molecules: a combined Raman, SERS and TERS study. J Raman Spectrosc. 2012;43:1895-904.

53. Buurmans ILC, Weckhuysen BM. Heterogeneities of individual catalyst particles in space and time as monitored by spectroscopy. Nat Chem. 2012;4:873-86. 
54. Kumar N, Stephanidis B, Zenobi R, Wain A, Roy D. Nanoscale mapping of catalytic activity using tip-enhanced Raman spectroscopy. Nanoscale. 2015;7:7133-7.

55. Domke KF, Pettinger B. In situ discrimination between axially complexed and ligand-free Co porphyrin on Au(111) with Tip-enhanced Raman spectroscopy. ChemPhysChem. 2009;10:1794-8.

56. Yeo BS, Amstad E, Schmid T, Stadler J, Zenobi R. Nanoscale probing of a polymer-blend thin film with Tip-enhanced Raman spectroscopy. Small. 2009;5:952-60.

57. Xue L, Li W, Hoffmann GG, Goossens JGP, Loos J, de With G. High-resolution chemical identification of polymer blend thin films using Tip-enhanced Raman mapping. Macromolecules. 2011;44:2852-8.

58. McNeill CR, Frohne H, Holdsworth JL, Furst JE, King BV, Dastoor PC. Direct photocurrent mapping of organic solar cells using a near-field scanning optical microscope. Nano Lett. 2004;4:219-23.

59. Wang X, Zhang D, Braun K, Egelhaaf HJ, Meixner AJ. Confocal and near-field spectroscopic investigation of P3HT:PCBM organic blend film upon thermal annealing. Plasmonics: Nanoimaging, Nanofabrication, and Their Applications V. 2009;7395:8.

60. Krebs FC, Norrman K. Analysis of the failure mechanism for a stable organic photovoltaic during $10000 \mathrm{~h}$ of testing. Prog Photovoltaics. 2007;15:697-712.

61. Zhang D, Wang X, Braun K, Egelhaaf HJ, Fleischer M, Hennemann L, et al. Parabolic mirror-assisted tip-enhanced spectroscopic imaging for non-transparent materials. J Raman Spectrosc. 2009:40:1371-6.

62. Cardona M. Light scattering in solids I. Berlin Heidelberg New York: Springer; 1983.

63. Poborchii V, Tada T, Kanayama T. Subwavelength-resolution Raman microscopy of Si structures using metal-particle-topped AFM probe. Japanese Journal of Applied Physics Part 2-Letters \& Express Letters. 2005;44:L202.

64. Saito Y, Motohashi M, Hayazawa N, Iyoki M, Kawata S. Nanoscale characterization of strained silicon by tip-enhanced Raman spectroscope in reflection mode. Appl Phys Lett. 2006;88:143109.

65. Hayazawa N, Motohashi M, Saito Y, Ishitobi H, Ono A, Ichimura T, et al. Visualization of localized strain of a crystalline thin layer at the nanoscale by tip-enhanced Raman spectroscopy and microscopy. J Raman Spectrosc. 2007;38:684-96.

66. Nguyen Q, Ossikovski R, Schreiber J. Contrast enhancement on crystalline silicon in polarized reflection mode tip-enhanced Raman spectroscopy. Opt Comm. 2007;274:231-5.

67. Ossikovski R, Nguyen Q, Picardi G. Simple model for the polarization effects in tip-enhanced Raman spectroscopy. Phys Rev B. 2007;75:045412.

68. Gucciardi PG, Lopes M, Deturche R, Julien C, Barchiesi D, de la Chapelle ML. Light depolarization induced by metallic tips in apertureless near-field optical microscopy and tip-enhanced Raman spectroscopy. Nanotechnology. 2008;19:215702.

69. Motohashi M, Hayazawa N, Tarun A, Kawata S. Depolarization effect in reflection-mode tip-enhanced Raman scattering for Raman active crystals. J Appl Phys. 2008;103:034309.

70. Saito Y, Motohashi M, Hayazawa N, Kawata S. Stress imaging of semiconductor surface by tip-enhanced Raman spectroscopy. J Microsc Oxford. 2008;229:217-22.

71. Hanafusa H, Hirose N, Kasamatsu A, Mimura T, Matsui T, Chong HMH, et al. Strain distribution analysis of sputter-formed strained Si by Tip-enhanced Raman spectroscopy. Appl Phys Express. 2011;4:025701.

72. Gucciardi PG, Bonaccorso F, Lopes M, Billot L, de la Chapelle ML. Light depolarization induced by sharp metallic tips and effects on Tip-Enhanced Raman Spectroscopy. Thin Solid Films. 2008;516:8064-72.

73. Welser J, Hoyt JL, Takagi S, Gibbons JF. Strain Dependence of the Performance Enhancement in Strained-Si N-MOSFETs. In: Int. Electron Devices Meeting Tech. Dig. San Francisco: IEEE; 1994. p. 373-6.

74. Thompson S, Anand N, Armstrong M, Auth C, Arcot B, Alavi M, et al. A $90 \mathrm{~nm}$ logic technology featuring 50nm strained silicon channel transistors, 7 layers of $C u$ interconnects, low k ILD, and 1 um $^{2}$ SRAM cell. In: Int. Electron Devices Meeting Tech. Dig. San Francisco: IEEE; 1994. p. 61-4.

75. Hermann P, Hecker M, Chumakov D, Weisheit M, Rinderknecht J, Shelaev A, et al. Imaging and strain analysis of nano-scale SiGe structures by tip-enhanced Raman spectroscopy. Ultramicroscopy. 2011;111:1630-5.

76. Domke KF, Pettinger B. Tip-enhanced Raman spectroscopy of $6 \mathrm{H}-\mathrm{SiC}$ with graphene adlayers: selective suppression of E1 modes. J Raman Spectrosc. 2009:40:1427-33.

77. Berweger S, Raschke MB. Polar phonon mode selection rules in tip-enhanced Raman scattering. J Raman Spectrosc. 2009;40:1413-9.

78. Berweger S, Neacsu CC, Mao Y, Zhou H, Wong SS, Raschke MB. Optical nanocrystallography with tip-enhanced phonon Raman spectroscopy. Nat Nanotechnol. 2009:4:496-9.

79. Matsui R, Verma P, Ichimura T, Inouye Y, Kawata S. Nanoanalysis of crystalline properties of GaN thin film using tip-enhanced Raman spectroscopy. Appl Phys Lett. 2007;90:061906.

80. Poliani E, Wagner MR, Reparaz JS, Mand M, Strassburg M, Kong X, et al. Nanoscale imaging of InN segregation and polymorphism in single vertically aligned InGaN/GaN multi quantum well nanorods by Tip-enhanced Raman scattering. Nano Lett. 2013;13:3205-12.

81. Cançado LG, Beams R, Jorio A, Novotny L. Theory of spatial coherence in near-field Raman scattering. Phys Rev X. 2014:4:031054

82. Hartschuh A, Sanchez EJ, Xie XS, Novotny L. High-resolution near-field Raman microscopy of single-walled carbon nanotubes. Phys Rev Lett. 2003;90:095503.

83. Hartschuh A, Anderson N, Novotny L. Near-field Raman spectroscopy using sharp metal Tip. J Microsc. 2003;210:234-40.

84. Anderson N, Hartschuh A, Cronin S, Novotny L. Nanoscale vibrational analysis of single-walled carbon nanotubes. J Am Chem Soc. 2005;127:2533-7.

85. Yano TA, Inouye Y, Kawata S. Nanoscale uniaxial pressure effect of a carbon nanotube bundle on tip-enhanced near-field Raman spectra. Nano Lett. 2006;6:1269-73.

86. Anderson N, Hartschuh A, Novotny L. Chirality changes in carbon nanotubes studied with near-field Raman spectroscopy. Nano Lett. 2007;7:577-82.

87. Maciel IO, Anderson N, Pimenta MA, Hartschuh A, Qian H, Terrones M, et al. Electron and phonon renormalization near charged defects in carbon nanotubes. Nat Mater. 2008;7:878-83. 
88. Yano T-a, Verma P, Saito Y, Ichimura T, Kawata S. Pressure-assisted tip-enhanced Raman imaging at a resolution of a few nanometres. Nat Photonics. 2009;3:473-7.

89. Peica N, Roehrig S, Ruediger A, Brose K, Thomsen C, Maultzsch J. Characterization of dye molecules and carbon nanostructures by tip-enhanced Raman spectroscopy. Phys Status Solidi B. 2009;246:2708-12.

90. Roy D, Wang J, Welland ME. Nanoscale imaging of carbon nanotubes using Tip enhanced Raman spectroscopy in reflection mode. Faraday Discuss. 2006;132:215-25.

91. Georgi C, Hartschuh A. Tip-enhanced Raman spectroscopic imaging of localized defects in carbon nanotubes. Appl Phys Lett. 2010;97:143117.

92. Roy D, Williams C. High resolution Raman imaging of single wall carbon nanotubes using electrochemically etched gold tips and a radially polarized annular beam. J Vac Sci Technol A. 2010;28:472-5.

93. Peica N, Thomsen C, Maultzsch J. Tip-enhanced Raman scattering along a single wall carbon nanotubes bundle. Phys Status Solidi B. 2010;247:2818-22.

94. Peica N, Thomsen C, Maultzsch J. Studying the local character of Raman features of single-walled carbon nanotubes along a bundle using TERS. Nanoscale Res Lett. 2011;6:1-7.

95. Goss K, Peica N, Thomsen C, Maultzsch J, Schneider CM, Meyer C. Index assignment of a carbon nanotube rope using tip-enhanced Raman spectroscopy. Phys Status Solidi B. 2011;248:2577-80.

96. Tarun A, Hayazawa N, Yano T-A, Kawata S. Tip-heating-assisted Raman spectroscopy at elevated temperatures. J Raman Spectrosc. 2011;42:992-7.

97. Weber-Bargioni A, Schwartzberg A, Cornaglia M, Ismach A, Urban JJ, Pang Y, et al. Hyperspectral nanoscale imaging on dielectric substrates with coaxial optical antenna scan probes. Nano Lett. 2011;11:1201-7.

98. Rauhut N, Engel M, Steiner M, Krupke R, Avouris P, Hartschuh A. Antenna-enhanced photocurrent microscopy on single-walled carbon nanotubes at $30 \mathrm{~nm}$ resolution. ACS Nano. 2012;6:6416-21.

99. Boehmler M, Hartschuh A. Tip-enhanced near-field optical microscopy of quasi-1 D nanostructures. ChemPhysChem. 2012;13:927-9.

100. Yano T-a, Ichimura T, Kuwahara S, H'Dhili F, Uetsuki K, Okuno Y, et al. Tip-enhanced nano-Raman analytical imaging of locally induced strain distribution in carbon nanotubes. Nat Commun. 2013;4:2592.

101. Roy D, Wang J, Williams C. Novel methodology for estimating the enhancement factor for Tip-enhanced Raman spectroscopy. J Appl Phys. 2009;105:013530.

102. Ferrari AC, Basko DM. Raman spectroscopy as a versatile tool for studying the properties of graphene. Nat Nanotechnol. 2013;8:235-46.

103. Marquestaut N, Talaga D, Servant L, Yang P, Pauzauskie P, Lagugne-Labarthet F. Imaging of single GaN nanowires by tip-enhanced Raman spectroscopy. J Raman Spectrosc. 2009:40:1441-5.

104. Boehmler M, Wang Z, Myalitsin A, Mews A, Hartschuh A. Optical imaging of CdSe nanowires with nanoscale resolution. Angew Chem Int Ed. 2011:50:11536-8.

105. Ogawa Y, Yuasa Y, Minami F, Oda S. Tip-enhanced Raman mapping of a single Ge nanowire. Appl Phys Lett. 2011:99:053112.

106. Geim AK, Grigorieva IV. Van der Waals heterostructures. Nature. 2013:499:419-25.

107. Pollard AJ, Kumar N, Rae A, Mignuzzi S, Su W, Roy D. Nanoscale optical spectroscopy: an emerging tool for the J Mater NanoSci. 2014;1:39-49.

108. Hoffmann GG, de With G, Loos J. Micro-Raman and tip-enhanced Raman spectroscopy of carbon allotropes. Macromol Symp. 2008;265:1-11.

109. Maximiano RV, Beams R, Novotny L, Jorio A, Cancado LG. Mechanism of near-field Raman enhancement in two-dimensional systems. Phys Rev B. 2012:85:235434

110. Stadler J, Schmid T, Zenobi R. Nanoscale chemical imaging of single-layer graphene. ACS Nano. 2011;5:8442-8.

111. Beams R, Cançado LG, Novotny L. Low temperature Raman study of the electron coherence length near graphene edges. Nano Lett. 2011;11:1177-81.

112. Snitka V, Rodrigues RD, Lendraitis V. Novel gold cantilever for nano-Raman spectroscopy of graphene. Microelectron Eng. 2011;88:2759-62

113. Weng S-W, Lin W-H, Su W-B, Hwu E-T, Chen P, Tsai T-R, et al. Estimating Young's modulus of graphene with Raman scattering enhanced by micrometer tip. Nanotechnology. 2014;25:255703.

114. Yampolsky S, Fishman DA, Dey S, Hulkko E, Banik M, Potma EO, et al. Seeing a single molecule vibrate through time-resolved coherent anti-Stokes Raman scattering. Nat Photonics. 2014;8:650-6.

115. Gross L, Mohn F, Moll N, Liljeroth P, Meyer G. The chemical structure of a molecule resolved by atomic force microscopy. Science. 2009;325:1110-4.

116. Weiss C, Wagner C, Kleimann C, Rohlfing M, Tautz FS, Temirov R. Imaging Pauli repulsion in scanning tunneling microscopy. Phys Rev Lett. 2010;105:086103.

117. Nie S, Emory SR. Probing single molecules and single nanoparticles by surface-enhanced Raman scattering. Science. 1997;275:1102-6.

118. Neacsu CC, Dreyer J, Behr N, Raschke MB. Scanning-probe Raman spectroscopy with single-molecule sensitivity. Phys Rev B. 2006;73:193406.

119. Zhang W, Yeo BS, Schmid T, Zenobi R. Single molecule Tip-enhanced Raman spectroscopy with silver tips. J Phys Chem C. 2007;111:1733-8.

120. Steidtner J, Pettinger B. High-resolution microscope for tip-enhanced optical processes in ultrahigh vacuum. Rev Sci Instrum. 2007;78:103104.

121. Steidtner J, Pettinger B. Tip-enhanced Raman spectroscopy and microscopy on single dye molecules with $15 \mathrm{~nm}$ resolution. Phys Rev Lett. 2008;100:236101.

122. Bortchagovsky E, Schmid T, Zenobi R. Internal standard for tip-enhanced Raman spectroscopy. Appl Phys Lett. 2013;103:043111.

123. Mukherjee S, Libisch F, Large N, Neumann O, Brown LV, Cheng J, et al. Hot electrons Do the impossible: Plasmon-induced dissociation of H-2 on Au. Nano Lett. 2013;13:240-7. 